\title{
ERD facility for analysis of hydrogen and deuterium in solids
}

\author{
T SOM, S DHAR, N BANERJI, K RAMAKRISHNAN and \\ V N KULKARNI \\ Department of Physics, Indian Institute of Technology, Kanpur 208016 , India
}

\begin{abstract}
Hydrogen is the lightest element in nature, and so, its detection and quantitatuve analysis is difficult by the conventional methods utilized for other elements. In the recent years the technique of elastic recoil detection analysis (ERD) using $1-2 \mathrm{MeV} \mathrm{He}^{+}$beam has been developed to quantitatively and simultaneously analyze hydrogen and its isotopes in solids. Such a facility has been set up using the $2 \mathrm{MeV}$ Van-de-Graaff accelerator at IIT Kanpur. It facilitates $\mathrm{H}$ and $\mathrm{D}$ analysis in a material up to a depth of $\sim 1 \mu \mathrm{m}$ with a detection sensitivity of (). 1 at. $\%$ and depth resolution of about $300 \AA$. The application potential of this setup is Illustrated by presenting the results of measurements performed on $\mathrm{Al}: \mathrm{H}: \mathrm{D}$ systems prepared by plasma source ion implantation and high $T_{\mathrm{C}}$ YBCO pellets exposed to humid atmosphere.
\end{abstract}

Keywords. Elastic recoil detection; hydrogen and deuterium incorporation; high- $T_{\mathrm{C}}$ superconductors: $\mathrm{Al}: \mathrm{H}$ and $\mathrm{Al}: \mathrm{D}$.

\section{Introduction}

The presence of hydrogen can have dramatic effects on the physical, chemical, mechanical and electrical properties of many materials. In the past two decades there has been an intense research activity to develop suitable techniques viz. nuclear reaction analysis (Lanford et al 1976), secondary ion mass spectroscopy (Magee and Wu 1978) and elastic recoil detection (ERD) (L'Ecuyer et al 1976; Doyle and Peercy 1979; Arnold Bik and Habraken 1993) for the quantitative analysis of hydrogen in the near surface region of materials. Among these, ERD is gaining more and more attention because it is non-destructive and has a good sensitivity and depth resolution. The measurement time in case of ERD is quite short (of the order of few tens of minutes). Further, it is possible to analyze hydrogen and its isotopes simultaneously. Although for ERD analysis heavy ions of energies $\sim 30-80 \mathrm{MeV}$ are used for detection of hydrogen and other light elements (L'Ecuyer et al 1978), $\mathrm{He}^{+}$beams obtained from low energy machines like 1-2 MeV Van-de-Graaff accelerators can also be utilized very effectively to analyze hydrogen and deuterium up to a depth of $\sim 1 \mu \mathrm{m}$ in a material. The purpose of this report is to describe the ERD facility which has been set up using the $2 \mathrm{MeV}$ Van-de-Graaff accelerator at the Department of Physics, IIT Kanpur. Results of the measurements performed on $\mathrm{Al}: \mathrm{H}, \mathrm{Al}: \mathrm{D}$ and $\mathrm{YBCO}: \mathrm{H}$ systems will be presented to illustrate the application potential of this facility.

\section{ERD set-up}

The accelerator (High Voltage Engineering Corporation (HVEC) make, USA, 1968) is currently being utilized for material analysis using the nuclear techniques, viz. Rutherford backscattering, channeling, proton induced X-ray emission and elastic recoil detection of hydrogen and deuterium. It is equipped with a RF ion source and ion beams of hydrogen, helium, argon, krypton and other gaseous species can be obtained 


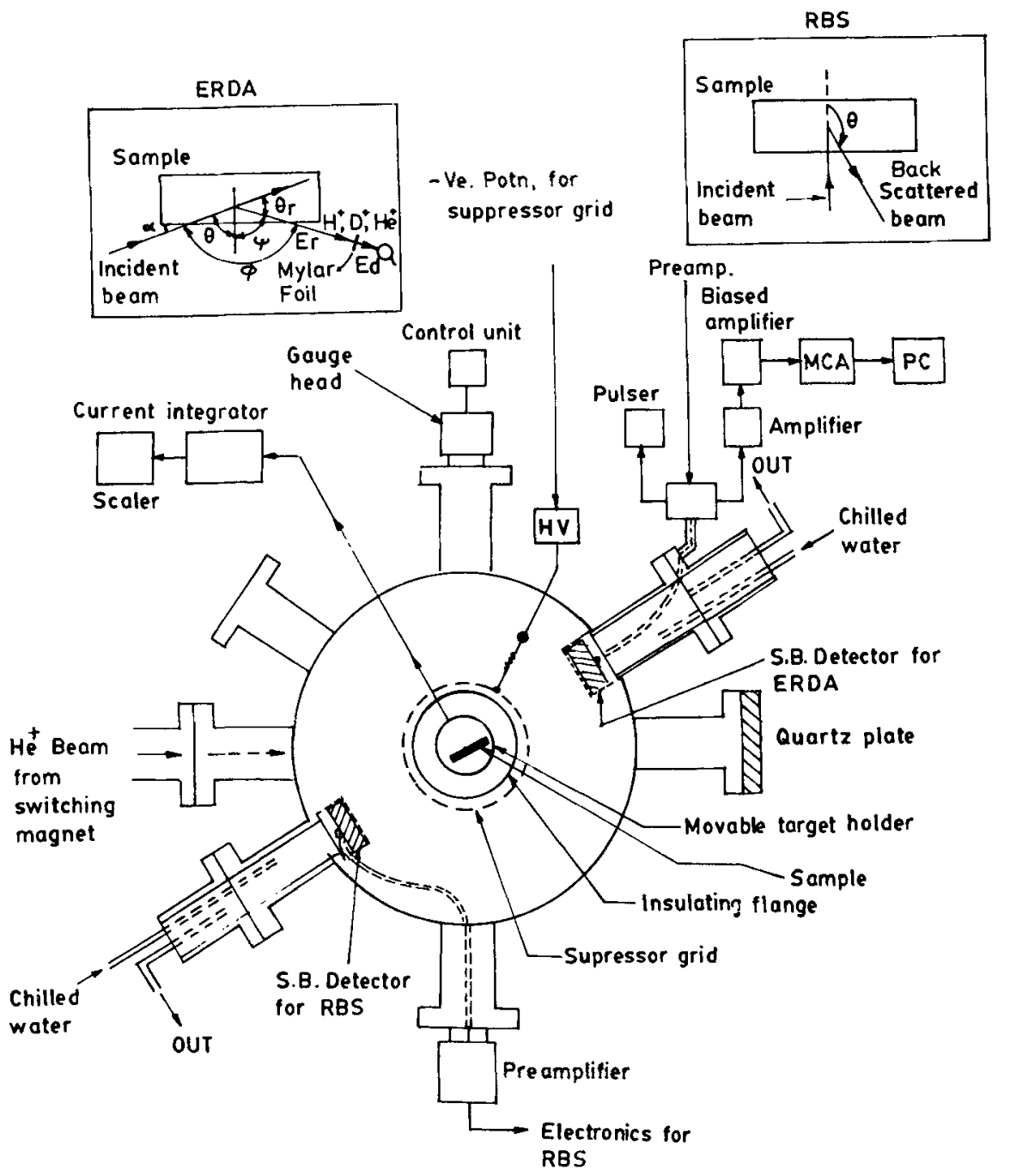

Figure 1. Block diagram of the ERD chamber and associated electronics. The chamber is also utilized for RBS and channeling experiments.

with a few microampere current on the target. The ion energy can be controlled to an accuracy of $\pm 1 \mathrm{keV}$. Magnetically analyzed $\mathrm{He}^{+}$beam in the energy range of 1.4 to 1.8 $\mathrm{MeV}$ is used for ERD analysis. The beam is collimated using different apertures along its path to obtain divergence of $<0.05^{\circ}$. The collimated beam enters the ERD chamber through adjustable beam defining slits. The details of the chamber are shown in figure 1. It is mounted on a diffstack pumping system equipped with a liquid nitrogen trap to perform experiments in a clean and high vacuum of $<5 \times 10^{-6}$ mbar. The important components of the ERD set-up are (i) holder for accurate positioning and orientation of the sample, (ii) secondary electron suppressor, (iii) arrangement to measure integrated charge, (iv) arrangement to mount and cool the silicon surface barrier detector and (v) mylar absorber to stop the forward scattered $\mathrm{He}^{+}$beam from reaching the 
ERD detector. The set-up can also be utilized for RBS and channeling analysis using a detector mounted at an angle of $150^{\circ}$ with respect to the beam direction. The ERD detector can accurately be positioned to make an angle varying from $20^{\circ}$ to $40^{\circ}$ with respect to the incident beam direction in a horizontal plane containing the incident beam. The aperture in front of the ERD detector has especially been designed to reduce the kinematical broadening. The signals from the detector are amplified using conventional nuclear electronics and are energy analyzed using a multichannel analyzer which is connected to a personal computer for data transfer.

\section{ERD formulism}

The scattering geometry of ERD is shown in the left top inset of figure 1. The quantitative analysis of hydrogen and deuterium mainly involves three basic concepts: (i) kinematic factor $(K)$, (ii) the differential recoil cross-section $(\mathrm{d} \sigma / \mathrm{d} \Omega$ ), and (iii) the stopping cross-sections of the incident and recoiled particles in the target.

The $\mathrm{He}^{+}$ion of incident energy $E_{0}$ recoils $\mathrm{H}$ and $\mathrm{D}$ from the surface whose energy is given by

$$
E_{\mathrm{r}}=K E_{0}
$$

where $K$ is the kinematic factor given by

$$
K=\left[4 M_{\mathrm{p}} M_{\mathrm{r}} /\left(M_{\mathrm{p}}+M_{\mathrm{r}}\right)^{2}\right] \cos ^{2} \theta_{\mathrm{r}}
$$

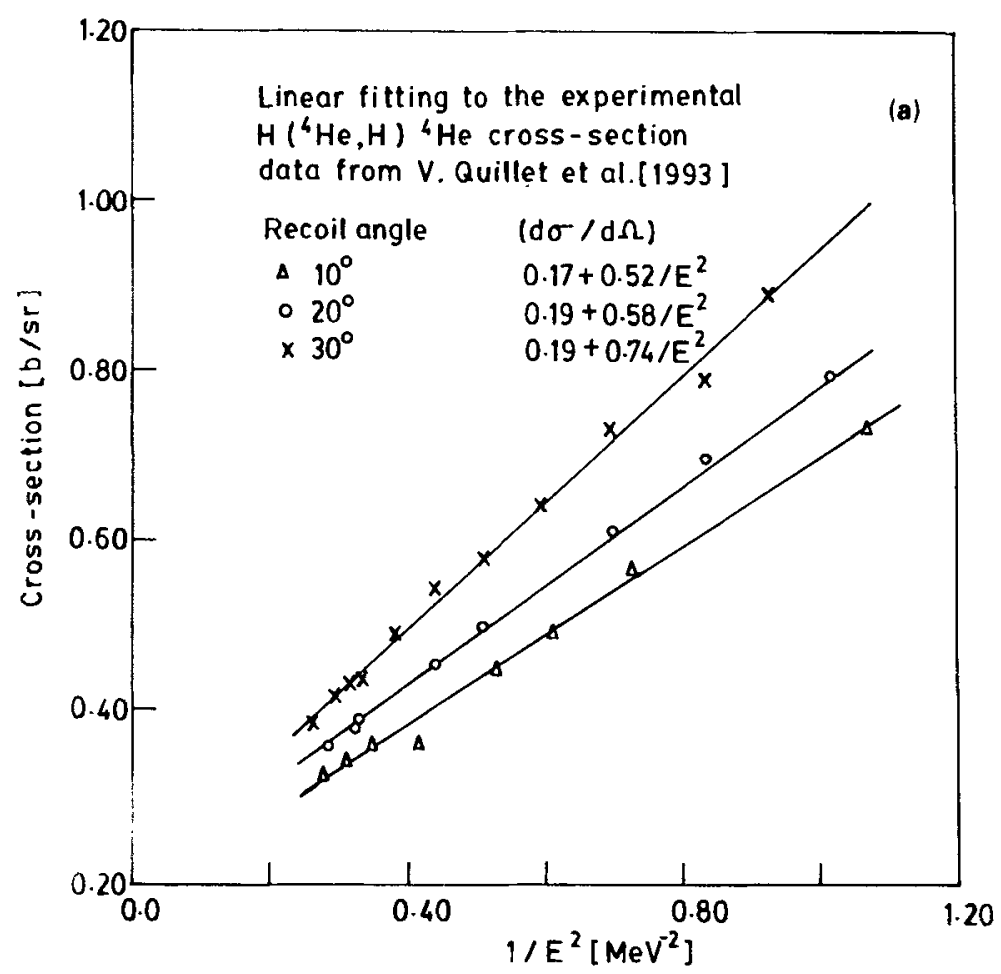

rigure 2. 


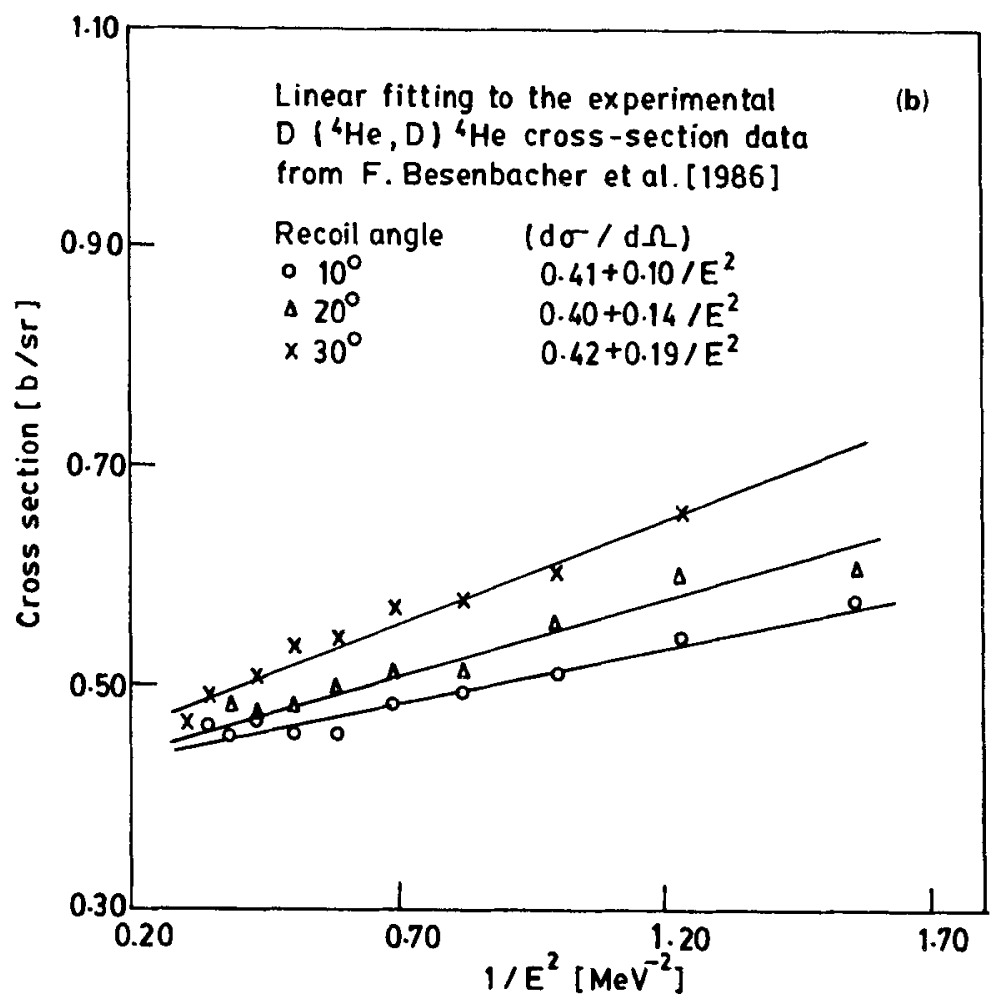

Figure 2. a. Linear fit to the experimental $\mathrm{H}\left({ }^{4} \mathrm{He}, \mathrm{H}\right){ }^{4} \mathrm{He}$ cross section data of Quillet et al (1993). Equations for recoil angles of $10^{\circ}, 20^{\circ}, 30^{\circ}$ are given in the figure. Cross-section values are in b/sr and energy is in $\mathrm{MeV}$ and b. Linear fit to the experimental D $\left({ }^{4} \mathrm{He}, \mathrm{D}\right){ }^{4} \mathrm{He}$ recoil cross section data of Besenbacher et al (1986).

where $M_{\mathrm{p}}$ and $M_{\mathrm{r}}$ are masses of the projectile and recoiled atoms respectively. Also incident helium ions get scattered in the forward direction due to the heavier matrix elements. These scattered primary ions are stopped from reaching the detector by the stopper foil of appropriate thickness. Assuming surface energy approximation (Doyle and Peercy 1979), the energy of $H$ or D getting recoiled from a depth $x$ within the sample is given by

$$
E_{\mathrm{d}}=K E_{0}-[S] x-E^{\prime}
$$

where $[S]$ is the energy loss factor given by

$$
[S]=\left\{K[\mathrm{~d} E / \mathrm{d} x]^{\mathrm{He}} /(\cos \theta)\right\}+\left\{[\mathrm{d} E / \mathrm{d} x]^{r} /\left(\cos \left(\theta+\theta_{\mathrm{r}}\right)\right\}\right.
$$

$[\mathrm{d} E / \mathrm{d} x]^{\mathrm{He}}$ is the stopping power for $\mathrm{He}^{+}$at its incident energy and $[\mathrm{d} E / \mathrm{d} x]^{r}$ the stopping power for either $\mathrm{H}$ or $\mathrm{D}$ at the surface recoiled energy $K E_{0}$ and $E^{\prime}$ the energy lost by the recoiled particles in the stopper foil. If the concentration (at./cc) of the recoiling species at a depth $x$ in the sample be $N(x)$ then

$$
N(x)=Y /\{Q(\mathrm{~d} \sigma / \mathrm{d} \Omega) \Omega(\delta E / R \sin \alpha[S])\},
$$

where $Y$ is the yield of recoils per channel at energy $E_{\mathrm{d}}, Q$ the total number of incident 
ions on the sample during the data acquisition time, $\delta E$ the detected energy window per channel, $R$ the ratio of the recoiled ion's stopping power in the absorber foil at energy $E_{\mathrm{r}}$ and $E_{\mathrm{d}}, \mathrm{d} \sigma / \mathrm{d} \Omega$ the elastic recoil cross section, $\Omega$ the solid angle subtended by the detector, and $x$ the grazing angle which the incident beam makes with the sample surface as shown in the inset of figure 1 . It should be noted that the recoil processes of hydrogen and deuterium from an incident $\mathrm{He}^{+}$ion of energy $\gtrsim 1 \mathrm{MeV}$ are not purely Coulombic in nature. Therefore, the cross-sections given by Rutherford formula are not valid in this case. Because of the application potential of ERD of $\mathrm{H}$ and D using helium ions there has been a renewed interest for measuring the $\mathrm{H}\left({ }^{4} \mathrm{He}, \mathrm{H}\right){ }^{4} \mathrm{He}$ and $\mathrm{D}\left({ }^{4} \mathrm{He}, \mathrm{D}\right){ }^{4} \mathrm{He}$ cross-section data accurately (Besenbacher et al 1986; Wang and Zhou 1988; Baglin et al 1992; Quillet et al 1993). The reported experimental recoil crosssection data for $\mathrm{H}$ and $\mathrm{D}$ are presented in figures $2 \mathrm{a}$ and $\mathrm{b}$ respectively. We have obtained linear fits to these data which have been used in the analysis. Several simulation codes namely RUMP (Doolittle 1985), SENRAS (Vizkelethy 1990), GISA3 (Saarilahti and Rauhala 1992) are now available for quick and accurate data analysis. We have installed the RUMP simulation code for the analysis of the recoil spectra.

From an experimental recoil spectrum, the depth versus concentration profile of the recoiled element in a sample can be obtained in an absolute manner. However, considering the uncertainties involved in the measurements of solid angle etc., it is necessary to standardize the set-up using a sample of known hydrogen content. We have used very good quality potassium dihydrogen phosphate $\left(\mathrm{KH}_{2} \mathrm{PO}_{4}\right.$ abbreviated as KDP) crystals for this purpose. The results of ERD of $\mathrm{H}$ from KDP is presented in the next section.

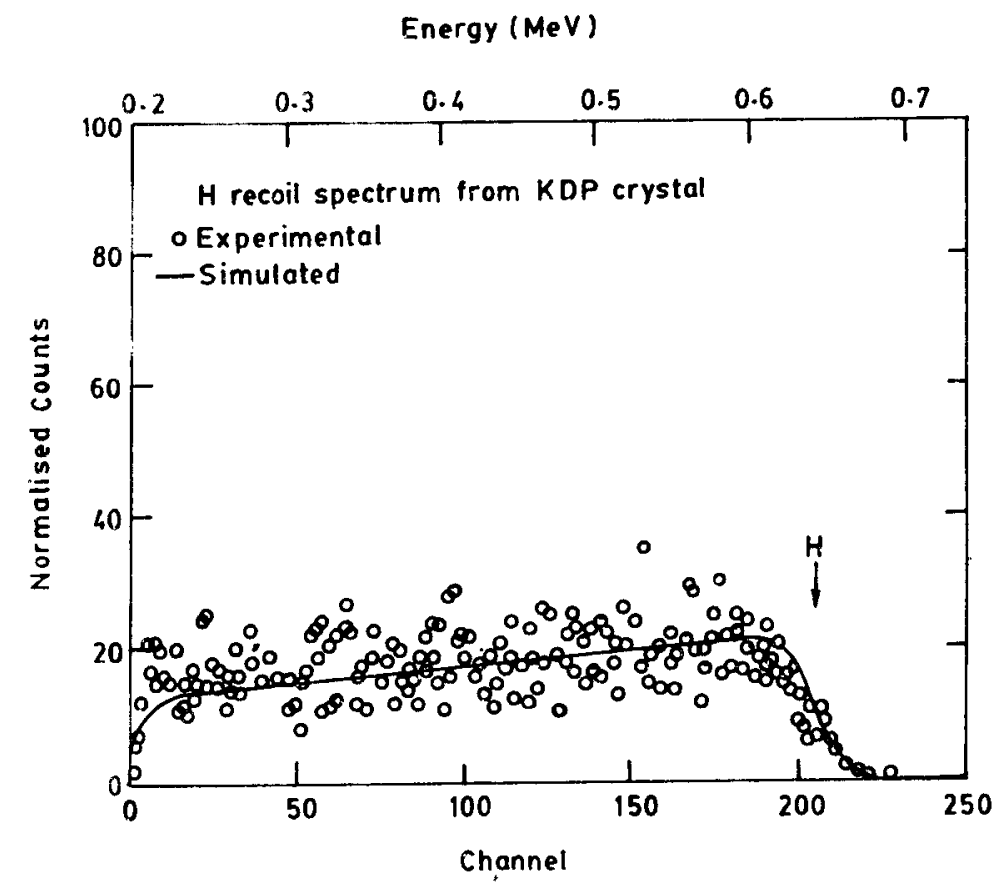

Figure 3. Recoil spectum of $\mathrm{H}$ from KDP crystal (open circles). The experimental parameters are given in the text. The arrow indicates the surface position of hydrogen. 


\section{ERD measurements on KDP crystals}

A typical spectrum of recoiled hydrogen from a KDP crystal, oriented at an angle of $15^{\circ}$ with respect to the incident beam direction, is shown in figure 3. This spectrum was taken using $\mathrm{He}^{+}$ions of energy $1.476 \mathrm{MeV}$, for an integrated charge of $3 \mu \mathrm{C}$, keeping the detector at an angle of $20^{\circ}$. The detector was covered with a mylar foil of thickness $6 \mu \mathrm{m}$ fixed on the aperture subtending a solid angle of $0.865 \mathrm{msr}$. The simulated spectrum shown by the solid line in figure 3 is generated by feeding the experimental parameters, the stoichiometry of KDP sample and the coefficients of the linear fit to the recoil cross-section data (figure $2 a$ ) in the RUMP code. The simulated spectrum agrees very well with the experimental spectrum. Assuming the hydrogen concentration to be uniform throughout the KDP sample, the overall energy resolution of the system is obtained from the initial rise of the recoil spectrum. It is found to be $35 \mathrm{keV}$ which corresponds to a depth resolution of about $300 \AA$. The maximum probed depth in this case is $0.5 \mu \mathrm{m}$. Detection sensitivity of $\mathrm{H}$ of 0.1 at. $\%$ in a material can be achieved by optimizing the $\mathrm{He}^{+}$energy, forward detection angle and the solid angle subtended by the detector. The probing depth of $\sim 1 \mu \mathrm{m}$ can be achieved with $2 \mathrm{MeV}$ incident $\mathrm{He}^{+}$ ions.

\section{Al:H and Al:D systems}

We now present a comparative study of annealing behaviour of $\mathrm{H}$ and $\mathrm{D}$ implanted in aluminium which has been performed using the above mentioned set-up. The system has been chosen because aluminium and its alloys have number of favourable properties which make them useful for vacuum systems and nuclear reactors. One of the important characteristics is the low rate of hydrogen permeation in these materials (Ichimura et al 1988).

Hydrogen and deuterium were incorporated in high purity polycrystalline aluminium samples by plasma source ion implantation (PSII) technique. The advantages of PSII relative to conventional ion implantation, are well described in literature (Conrad et al 1987). It is a very cost-effective technique and more compatible to 'in-house' operation as opposed to the other ion beam processing instruments.

Two sets of samples were prepared. Samples of set $1(\mathrm{Al}: \mathrm{H})$ were implanted at $1 \mathrm{keV}$ with only hydrogen. Samples of set 2 (Al:H:D) were first implanted with $1 \mathrm{keV}$ hydrogen and subsequently with deuterium at $3 \mathrm{keV}$. The ERD analysis was performed immediately after implantation. A typical spectrum of as implanted $\mathrm{Al}: \mathrm{H}$ sample is shown in figure 4 . The area under the hydrogen profile gives the total quantity of hydrogen of $5 \cdot 8 \times 10^{16}$ atoms $/ \mathrm{cm}^{2}$ in the sample. The simulation analysis shows that $750 \AA$ thick layer having composition of $\mathrm{Al}_{1} \mathrm{H}_{0.65}$ is formed on the surface of the aluminium sample. The ERD spectra obtained from the $\mathrm{Al}$ : $\mathrm{H}$ sample after annealing at $200^{\circ} \mathrm{C}$ for $1 \mathrm{~h}$ and $3 \mathrm{~h}$ are compared with the spectrum of the as implanted sample in figure 5 . There is no change in the $\mathrm{H}$ profile after $1 \mathrm{~h}$ of annealing while a small reduction in the hydrogen content is seen to occur after $3 \mathrm{~h}$ of annealing. This indicates that a stable Al--H phase has been formed after implantation. However, this has to be confirmed by TEM or X-ray measurements.

The recoil spectra of $\mathrm{Al}: \mathrm{H}: \mathrm{D}$ in the as prepared condition and after annealing are shown in figure 6 . The $H$ and $D$ peaks are well separated in the spectrum which enables 
Energy (MeV)

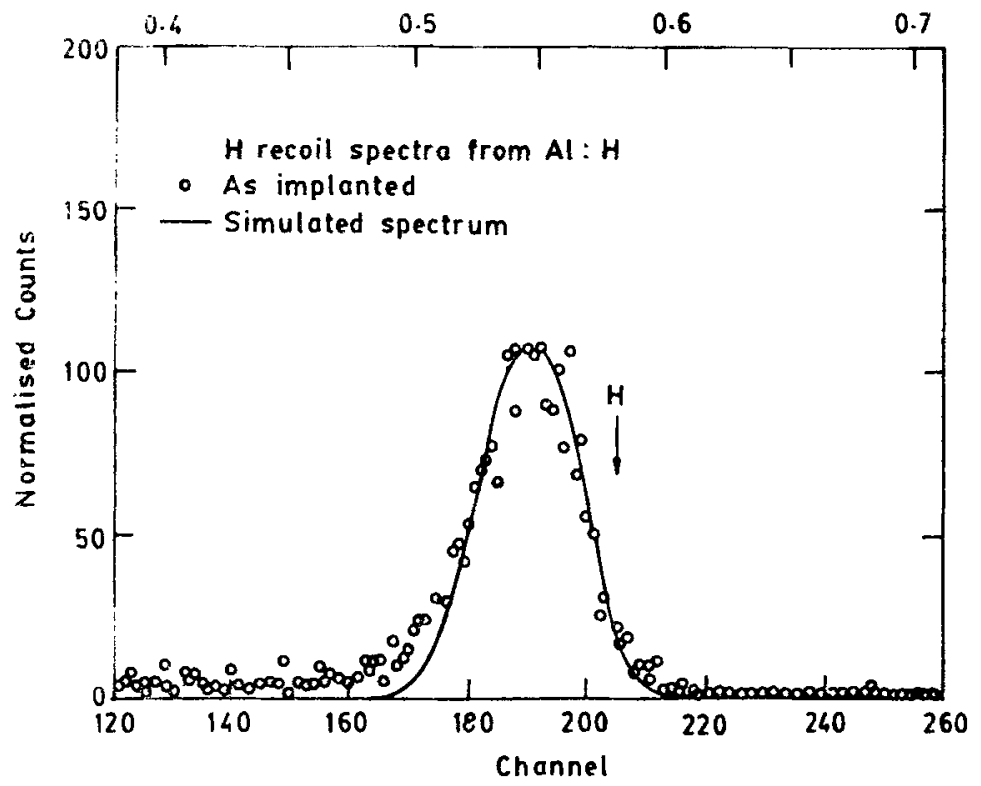

Figure t. H iccoil spectrum (open circles) from Al:H sample after implantation. For this spectrum the experimental parameters are: $E_{0}=1.7 \mathrm{MeV}, Q=10 \mu \mathrm{C}, \Omega=0.468 \mathrm{msr}$ and $1)_{r}=30$. The arrow indicates the surface position of hydrogen. The simulated curve (solid line) 1s obtained using a $700 \AA$ layer of composition $\mathrm{Al}_{1} \mathrm{H}_{0.65}$.

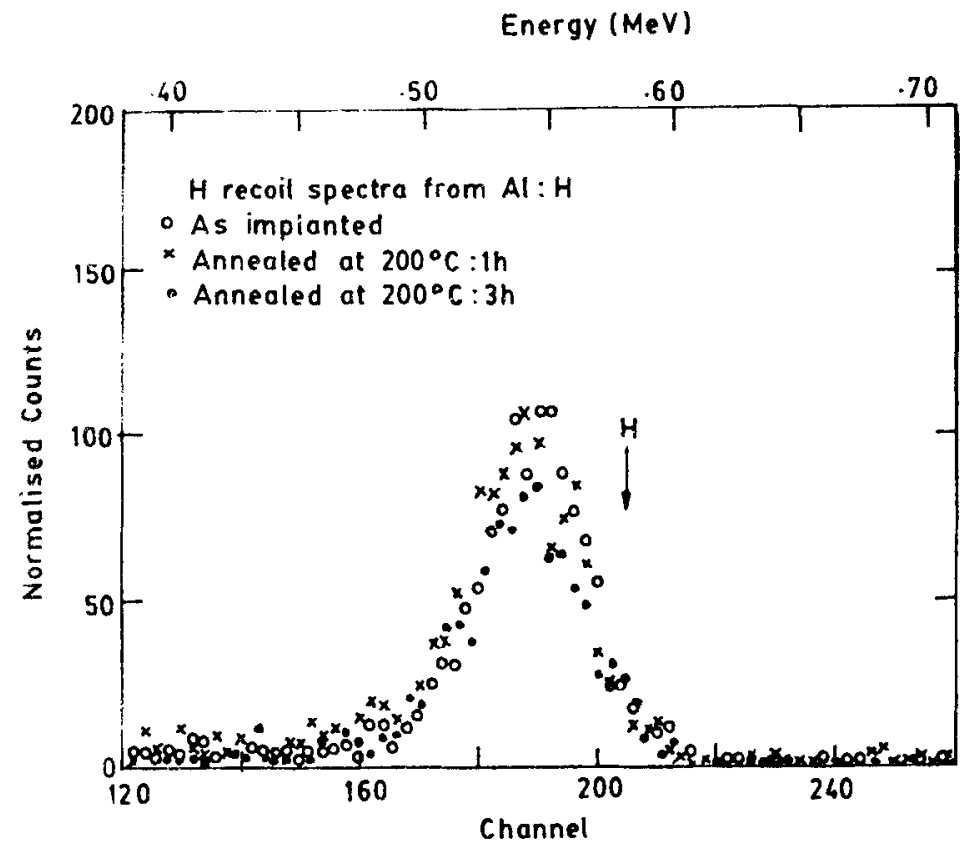

Higure 5. Comparison of $\mathrm{H}$ recoul specira of $\mathrm{Al}: \mathrm{H}$ sample before and after annealing at $200^{\circ} \mathrm{C}$ for $1 \mathrm{~h}$ and $3 \mathrm{~h}$. Experimental parameters are same as in figure 4 . 


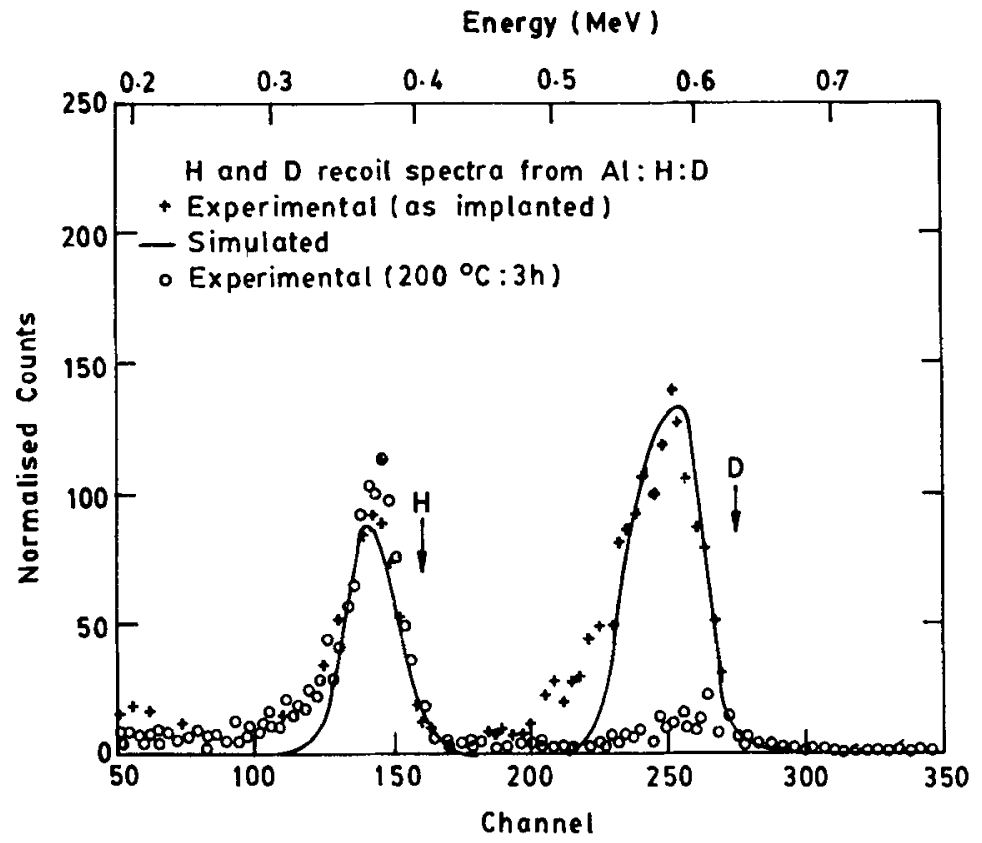

Figure 6. $\mathrm{H}$ and $\mathrm{D}$ recoil spectra from $\mathrm{Al}: \mathrm{H}: \mathrm{D}$ sample in the as-implanted condition ( + ) and after annealing $(O)$ at $200^{\circ} \mathrm{C}$ for $3 \mathrm{~h}$. The arrows indicate the surface positions of $\mathrm{H}$ and $\mathrm{D}$. The simulated spectrum for as-implanted case is shown by the solid line. Experimental parameters are same as in figure 4.

one to analyze both $\mathrm{H}$ and $\mathrm{D}$ simultaneously, using helium beam as a probe for ERD. The implanted area under $H$ and $D$ signals yield total amount of $8.02 \times 10^{17}$ and $3.34 \times 10^{18}$ at. $/ \mathrm{cm}^{2}$ of $\mathrm{H}$ and $\mathrm{D}$ respectively. The simulation gives two layers having compositions $\mathrm{Al}_{1} \mathrm{H}_{0.25} \mathrm{D}_{0.35}$ of thickness $400 \AA$ and $\mathrm{Al}_{1} \mathrm{H}_{0.82} \mathrm{D}_{0.25}$ of thickness $700 \AA$ respectively on the bulk aluminium sample. After annealing, the $\mathrm{H}$ profile does not show any significant change but interestingly a drastic reduction $(\sim 99 \%)$ in the amount of $\mathrm{D}$ is observed. This indicates that deuterium atoms have not formed phase with $\mathrm{Al}: \mathrm{H}$ system obtained by implanting $\mathrm{H}$ first and therefore $\mathrm{D}$ is released from the sample after heating. This phenomenon is being studied in detail.

\section{YBCO:H system}

The incorporation of hydrogen in superconductors has been reported to alter several of its properties, such as, structural changes (Fujii et al 1988), dependence on $T_{\mathrm{C}}$ values (Reilly et al 1987), magnetic ordering (Niedermayer et al 1989) etc. Since hydrogen can get incorporated in the superconducting material via absorption of water vapour from the atmosphere, we have exposed the as prepared pellet to a humid atmosphere and then analyzed it by ERD. The $\mathrm{H}$ recoil spectra from YBCO pellets in as prepared condition and after exposing it to the humid atmosphere (for $3.5 \mathrm{~h}$ ) are shown in figure 7 . The hydrogen concentration in the as prepared sample was below the ERD detection limit $(<0 \cdot 1$ at. $\%)$. Analysis of the ERD spectrum of the exposed sample shows that a layer of thickness $>0.5 \mu$ m having a composition of $\mathrm{H}_{1.2} \mathrm{Y}_{1} \mathrm{Ba}_{2} \mathrm{Cu}_{3} \mathrm{O}_{6.8}$ 


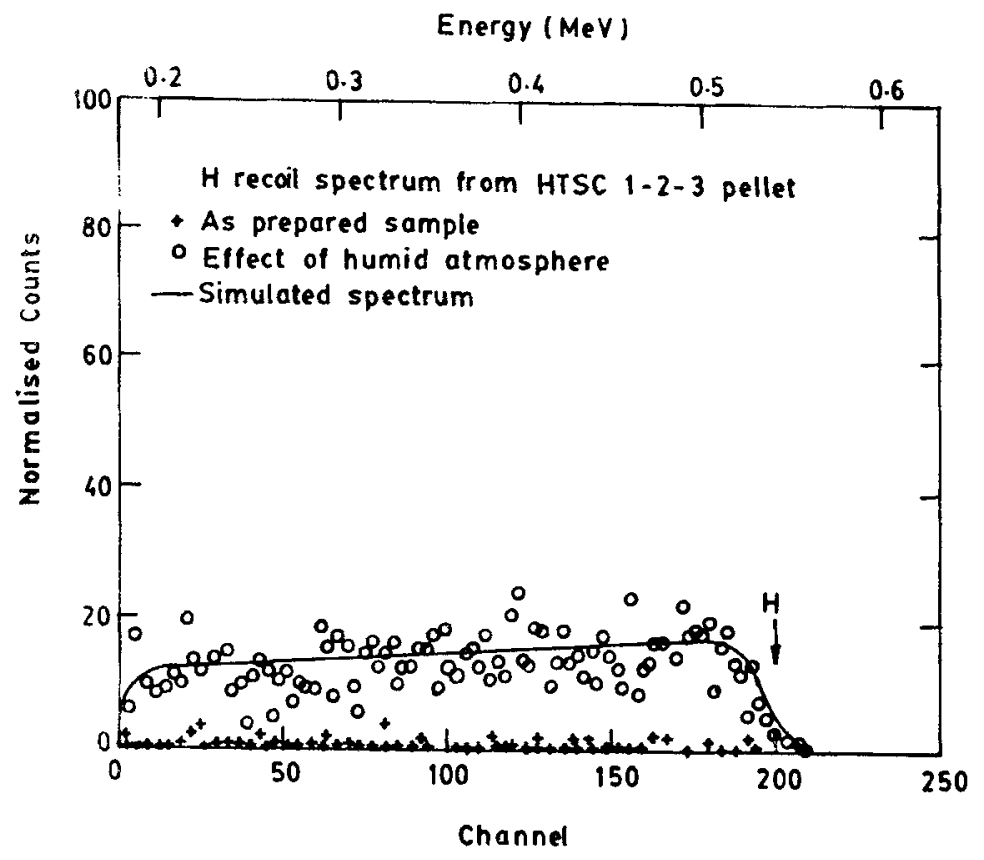

Figure 7. Expermental $H$ recoll spectra from high- $T_{C} Y B C O$ pellet before $(+)$ and after exposure to humid atmosphere $(\mathrm{O})$. The experimental parameters are: $E_{0}=1.427 \mathrm{MeV}$, $Q=10 \mu \mathrm{C}, \Omega=0.865 \mathrm{msr}$ and $\theta_{\mathrm{r}}=25^{\circ}$. The arrow indicates the surface position of recoiled hydrogen. The simulated spectrum for $(O)$ is obtained by using a composition of $\mathrm{H}_{1.2} \mathrm{Y}_{1} \mathrm{Ba}_{2} \mathrm{Cu}_{3} \mathrm{O}_{68}$ of thickness $>0.5 \mu \mathrm{m}$ and is indicated by the solid line.

has been formed in the pellet. A systematic study of the absorption of hydrogen and its effects on the physical properties of the system are underway.

\section{Summary and conclusion}

The ERD facility to analyze hydrogen and deuterium in materials has been set up using the $2 \mathrm{MeV}$ Van-de-Graaff accelerator at IIT Kanpur. With this set-up hydrogen and deuterium can simultaneously be analyzed in a material up to a depth of $\sim 1 \mu \mathrm{m}$ with sensitivity of $0 \cdot 1$ at. \% and with depth resolution of $\sim 300 \AA$.

Implanted hydrogen in polycrystalline aluminium appears to form a phase which is stable up to $200^{\circ} \mathrm{C}$. On the other hand, deuterium implanted in previously formed $\mathrm{Al}: \mathrm{H}$ phase gets released from the surface after annealing.

Exposure of as prepared high- $T_{\mathrm{C}}$ superconducting pellets to the humid atmosphere for a few hours results in a hydrogen absorption up to 8.5 at. $\%$.

\section{Acknowledgements}

The authors would like to thank Prof. R M Singru, Department of Physics, IIT Kanpur for encouragement and valuable suggestions. We would like to thank Prof. M S Hegde, Department of Chemistry, IISC Bangalore, for suggesting KDP sample for ERD 
experiments. We thank the technical personnels of the Central Nuclear Laboratories, Department of Physics, IIT Kanpur. Financial support by the Department of Science and Technology (DST) is gratefully acknowledged.

\section{References}

Arnold Bik W M and Habraken F H P M 1993 Rep. Prog. Phys. 56859

Baglin J E E, Kellock A J, Crockett M A and Shih A H 1992 Nucl. Instrum. \& Meth. B64 469

Besenbacher F, Stensgaard I and Vase P 1986 Nucl. Instrum \& Meth. B15 459

Conrad J R, Radtke J L, Dodd R A, Worzala Frank J and Tran Ngoc C 1987 J. Appl. Phys. 624591

Doolittle L R 1985 Nucl. Instrum \& Meth. B9 334

Doyle B L and Peercy P S 1979 Appl. Phys. Lett. 34811

Fujii H, Kawanaka H, Ye W. Orimo S and Fukuba H $1988 \mathrm{Jpn}$ J. Appl. Plyss. 27 L525

Ichimura M, Katsuta H, Sasajima Y and Imabayashi M 1988 J. Phys. Chem. Solids 491259

Lanford W A, Trautvetter H P, Zeigler J F and Keller J 1976 Appl. Phys. Lett. 28566

L'Ecuyer J, Brassard C and Cardinal C 1978 Nucl. Instrum. \& Meth. 149271

Magee C W and Wu C P 1978 Nucl. Instrum. \& Meth. 149529

Nicdermayer Ch, Gluckler H, Simon R, Golnik A, Rauer M, Recknagel E, Weidinger A, Budnick I I, Paulus W and Schollhorn R 1989 Phys. Rev. B40 11386

Quillet V, Abel F and Schott M 1993 Nucl. Instum. \& Meth. B83 47

Reilly J J, Suenaga M, Johnson J R, Thompson P and Moodenbaugh A R 1987 Phys. Rev. B36 5694

Saarilahti J and Rauhala E 1992 Nucl. Instrum. \& Meth. B64 734

Vizkelethy G 1990 Nucl. Instrum. \& Meth. B45 1

Wang H and Zhou G Q 1988 Nucl. Instrum. \& Meth. B34 145 